\title{
A Generalization of the Radon-Nikodym Property in Dual Banach Spaces, Fragmentedness, and Differentiability of Convex Functions
}

By

\author{
Minoru MatsudA*
}

\begin{abstract}
For non-empty bounded subsets $A$ of Banach spaces, we introduce the notion of the $A$ Radon-Nikodym property in dual Banach spaces, a slight generalization of the Radon-Nikodym property in such spaces. Making the effective use of this notion and a weak*-measurable function constructed here, we give a direct study of some related properties (especially, $A$-fragmentedness) of weak*-compact subsets of dual Banach spaces.
\end{abstract}

\section{§1. Introduction}

Throughout this paper, $X$ denotes an arbitrary real Banach space, $X^{*}$ its topological dual space and $B(X)$ the closed unit ball of $X$. In the following, $(S, \Sigma, \mu)$ always denotes a complete finite measure space and $(I, \Lambda, \lambda)$ is the Lebesgue measure space on $I(=[0,1])$. We always understand that $I$ is endowed with $\Lambda$ and $\lambda$. For each $g \in L_{\infty}(S, \Sigma, \mu)$ and $E \in \Sigma^{+}(=\{E \in \Sigma$ : $\mu(E)>0\}$ ), ess- $0(g \mid E)$ denotes the essential oscillation of $g$ (as a function) on $E$, and for each $E \in \Sigma^{+}$, denote $\Delta(E)=\left\{\chi_{F} / \lambda(F): F \subset E, F \in \Sigma^{+}\right\}$. For each $(S, \Sigma, \mu)$, a function $f: S \rightarrow X^{*}$ is said to be weak*-measurable if the realvalued function $(x, f(s))$ is $\mu$-measurable for each $x \in X$. If $f: S \rightarrow X^{*}$ is a bounded weak*-measurable function, we obtain a bounded linear operator $U_{f}$ : $X \rightarrow L_{\infty}(S, \Sigma, \mu) \quad\left(\right.$ resp. $\quad T_{f}: X \rightarrow L_{1}(S, \Sigma, \mu)$ ) given by $U_{f}(x)=x \circ f$ (resp. $\left.T_{f}(x)=x \circ f\right)$ for every $x \in X$, where $(x \circ f)(s)=(x, f(s))$ for every $s \in S$. The dual operator of $T_{f}$ is denoted by $T_{f}^{*}\left(: L_{\infty}(S, \Sigma, \mu) \rightarrow X^{*}\right)$. Furthermore,

Communicated by Y. Takahashi, July 8, 1999.

1991 Mathematics Subject Classification(s): 46B22, 46G10, 46B07, 46B20.

* Department of Mathematics, Faculty of Science, Shizuoka University, Ohya, Shizuoka 422-8529, Japan. 
if we define a vector measure $\alpha_{f}$ (associated with such a function $f$ ) : $\Sigma \rightarrow X^{*}$ by $\alpha_{f}(E)=T_{f}^{*}\left(\chi_{E}\right)$ for every $E \in \Sigma$, we then have that

$$
\left(x, \alpha_{f}(E)\right)=\int_{E}(x, f(s)) d \mu(s)
$$

for every $x \in X$ and every $E \in \Sigma$. When a vector measure $\alpha: \Sigma \rightarrow X^{*}$ satisfies that $\alpha(E)=T_{f}^{*}\left(\chi_{E}\right)$ for every $E \in \Sigma$, we say that it has the weak*-density $f$.

Now, for weak*-compact sets in dual Banach spaces, we have the notion of Radon-Nikodym sets ( $\mathrm{RN}$ sets in brief), which is a generalization of weak*compact convex sets with the Radon-Nikodym property ( $R N P$ in brief). The notion of RN sets has been defined and studied in Reynov [12]. Succeedingly, Fitzpatrick [3] has defined the notion of separably related sets in $X^{*}$ as a generalization of RN sets, and he has made a study of them. In this paper, in order to analyze such various notions and related properties in a unified manner, we wish to introduce the notion of the $A$-RNP which is more general than that of the RNP in dual Banach spaces and to give attention especially to a continuity property of certain maps (that is, bare continuity of the identity map, cf. [10]). For that, let us define following notions. They are slight generalizations of fragmentedness (cf. [4]), strong measurability for weak*measurable functions and the RNP in dual Banach spaces, respectively.

Definition 1 . Let $A$ be a bounded subset of $X$ and $K$ a weak*-compact (not necessarily convex) subset of $X^{*}$. Then $K$ is said to be $A$-fragmented if every weak*-compact subset $D$ of $K$ has the following property: For every $\varepsilon>0$, there exists a weak*-open subset $U$ such that $U \cap D \neq \varnothing$ and $\operatorname{diam}_{A}(U \cap D)\left(=\sup \left\{q_{A}\left(u^{*}-v^{*}\right): u^{*}, v^{*} \in U \cap D\right\}\right)<\varepsilon$.

Here $q_{A}$ is the seminorm given by $q_{A}\left(x^{*}\right)=\sup _{x \in A}\left|\left(x, x^{*}\right)\right|$ for every $x^{*} \in X^{*}$.

Note that if $A=B(X)$ in Definition 1, we have the usual notion of fragmentedness for weak*-compact subsets of $X^{*}$.

Definition 2. Let $A$ be a bounded subset of $X$.

(1) Let $f: S \rightarrow X^{*}$ be a function. Then $f$ is said to be $A$-strongly measurable if $f$ has the following two conditions.

(a) $f$ is weak*-measurable.

(b) For every $\varepsilon>0$ and $E \in \Sigma^{+}$, there exists $F \in \Sigma^{+}$with $F \subset E$ such that $\operatorname{diam}_{A}(f(F))\left(=\sup _{x \in A} 0(x \mid f(F))\right)<\varepsilon$.

(2) Let $f: S \rightarrow X^{*}$ be a weak*-measurable function. Then $f$ is said to have the $A$-strongly measurable decomposability if there exists an $A$-strongly measurable function $g: S \rightarrow X^{*}$ such that $f-g$ is weak*-scalarly null (that is, $(x, f(s)-g(s))=0 \mu$-a.e. on $S$ for every $x \in X)$. 
Definition 3. Let $A$ be a bounded subset of $X$ and $K$ a weak*-compact convex subset of $X^{*}$. Then the set $K$ is said to have the $A$-RNP with respect to $(S, \Sigma, \mu)$ if for any vector measure $\alpha: \Sigma \rightarrow X^{*}$ for which $\alpha(E) \in \mu(E) \cdot K$ for every $E \in \Sigma$, there exists an $A$-strongly measurable function $f: S \rightarrow K$ such that $\alpha(E)=T_{f}^{*}\left(\chi_{E}\right)$ for every $E \in \Sigma$ (that is, any such vector measure $\alpha$ has an $A$ strongly measurable weak ${ }^{*}$-density $f$ valued in $K$ ). The set $K$ is said to have the $A$-RNP if $K$ has this property with respect to all complete finite measure spaces.

Note that if $A=B(X)$ in Definition 3, we have the usual notion of the RNP for weak*-compact convex subsets of $X^{*}$.

Well, in a series of our papers [6], [7], [8] and [9], we have made a study of $K$-weakly precompact sets $A$ in Banach spaces by the effective use of a kind of dentability and $K$-valued weak*-measurable functions constructed in the case where $A$ is non- $K$-weakly precompact. Our approach to such notions is different from others in the meaning that its process is independent of results already obtained when $A=B(X)$ or $K=B\left(X^{*}\right)$, and the focus is on the construction of $K$-valued weak*-measurable functions with various desired properties.

In this paper as well, by following the ideas of the best use of another kind of dentability and a basic $K$-valued weak*-measurable function constructed in the case where $K$ is non- $A$-fragmented, we wish to clarify certain kinds of structure of weak*-compact $A$-fragmented sets in a slightly direct process (that is, from a standpoint to deal with a weak*-compact $A$-fragmented set itself). Then we have the following theorem, which is the main result of this paper. For notations and terminology used (and not explained) in Theorem, see $\S 2$.

Theorem. Let $A$ be a bounded subset of $X$ and $K$ a weak*-compact subset of $X^{*}$. Then the followng statements about $A$ and $K$ are equivalent.

(1) The set $\overline{\mathrm{co}}^{*}(K)$ (the weak*-closed convex hull of $K$ ) has the $A-R N P$.

(2) The set $\overline{\mathrm{co}}^{*}(K)$ has the $A-R N P$ with respect to $(I, \Lambda, \lambda)$.

(3) For every weak*-measurable function $f: I \rightarrow K, U_{f}(A)$ is equimeasurable in $L_{\infty}(I, \Lambda, \lambda)$.

(4) The set $K$ is A-fragmented.

(5) The set $\overline{\mathrm{co}}^{*}(K)$ is $A$-fragmented.

(6) Every weak ${ }^{*}$-compact convex subset of $\overline{\mathrm{co}}^{*}(K)$ is $A$-weak*-dentable.

(7) For every continuous convex function $g: X \rightarrow R$ such that $\partial g(x) \subset$ $\overline{\mathrm{co}}^{*}(K)$ for all $x \in X$, there exists a dense $G_{\delta}$-subset $G$ of $X$ such that $g$ is $\operatorname{aco}(A)$ (the absolutely convex hull of $A$ )-differentiable at each $x \in G$.

(8) If $\left\{z_{n}\right\}_{n \geqq 1}$ is a sequence in $A$ and $H$ is a non-empty subset of $K$, then there is a point $x \in X$ such that $c_{H}$ is $\Psi$-uniformly Gateaux differentiable at $x$, where $\Psi=\left\{z_{n}: n \geqq 1\right\}$. 
Needless to say, some parts of Theorem may be well-known essentially. But, some parts are exactly new, and the points to be emphasized in Theorem are that implications (2) (or (3)) $\Rightarrow(4),(6) \Rightarrow(1)$ and $(8) \Rightarrow(4)$ and their proofs can be given directly. Further, we wish to note two things. One is the importance of the notion of $A$-fragmentedness acting as intermediary in our consideration. The other is that we can appreciate the notion of the $A$-RNP in dual Banach spaces by combining with a basic weak*-measurable function constructed here concretely.

The paper is organized as follows. In $\S 2$, we give some more definitions and preliminary results. In $\S 3$, we present a basic $K$-valued weak*-measurable function associated with a weak*-compact non- $A$-fragmented set $K$ and give a complete proof of Theorem, making use of results in $\S 2$ and this section. Finally, in $\S 4$, we give some remarks on Theorem.

\section{§2. Preliminary Results}

If $g: X \rightarrow R$ is a continuous convex function, for $x, y \in X$, we define $D g(x, y)$ by

$$
\lim _{t \rightarrow 0}\{g(x+t y)-g(x)\} / t
$$

provided that this limit exists. We say that $g$ is Gateaux differentiable at $x \in X$ if $D g(x, y)$ exists for every $y \in X$. We define the subdifferential of $g$ at $x(\in X)$ to be the set $\partial g(x)$ of all elements $x^{*}$ of $X^{*}$ satisfying that $\left(u, x^{*}\right) \leqq g(x+u)-$ $g(x)$ for any $u \in X$. Then $\partial g(x)$ is a non-empty weak*-compact convex subset of $X^{*}$ for every $x \in X$. Further, we give:

Definition 4. Let $g: X \rightarrow R$ be a continuous convex function and $A$ a bounded subset of $X$. Then

(1) ([1]). $g$ is said to be $A$-differentiable at $x \in X$ if there exists $x^{*} \in X^{*}$ such that

$$
\lim _{t \rightarrow 0+}\left\{\sup _{y \in A}\left|(g(x+t y)-g(x)) / t-\left(y, x^{*}\right)\right|\right\}=0 .
$$

If $A=B(X), g$ is said to be Frechet differentiable at $x \in X$.

(2) $g$ is said to be $A$-uniformly Gateaux differentiable at $x \in X$ if $D g(x, y)$ exists uniformly in $y \in A$.

In order to analyze weak*-compact $A$-fragmented sets from our view-point, we turn our attention especially to continuous convex functions defined as follows.

Definition 5. Let $H$ be a non-empty bounded subset of $X^{*}$. Then the continuous convex function associated with $H$, which is denoted by $c_{H}$, is 
defined by

$$
c_{H}(x)=\sup _{x^{*} \in H}\left(x, x^{*}\right)
$$

for each $x \in X$. This function $c_{H}$ is called the support function of $H$ ([11]).

Then the fact that $\partial c_{H}(x) \subset \overline{\mathrm{co}}^{*}(H)$ for every $x \in X$ is well-known. And in order to check the $A$-uniform Gateaux differentiability of continuous convex functions, the following well-known result is useful later.

Proposition 1. Let $g: X \rightarrow R$ be a continuous convex function and $A$ a bounded subset of $X$. Then

(1) $g$ is A-uniformly Gateaux differentiable at $x \in X$ if and only if $g$ satisfies the following:

$$
\begin{aligned}
& \lim _{t \rightarrow 0+}\left\{\sup _{y \in A}(g(x+t y)+g(x-t y)-2 g(x)) / t\right\} \\
& \left(=\inf _{t>0}\left\{\sup _{y \in A}(g(x+t y)+g(x-t y)-2 g(x)) / t\right\}\right)=0 .
\end{aligned}
$$

(2) $g$ is $B(X)$-uniformly Gateaux differentiable at $x \in X$ if and only if $g$ is Frechet differentiable at $x \in X$.

In order to see geometric properties of weak*-compact convex subsets with the $A$-RNP, we introduce the notion of $A$-weak*-dentability for bounded subsets of dual Banach spaces ([3]).

Definition 6. (1) Let $H$ be a non-empty bounded subset of $X^{*}$. A weak*open slice of $H$ is a set of the form:

$$
S(x, c, H)=\left\{x^{*} \in H:\left(x, x^{*}\right)>\sup _{z^{*} \in H}\left(x, z^{*}\right)-c\right\}
$$

where $x \in X$ and $c>0$.

(2) Let $A$ be a bounded subset of $X$ and $H$ a bounded subset of $X^{*}$. Then the set $H$ is said to be $A$-weak*-dentable if $H$ has weak*-open slices of arbitrarily small $q_{A}$-diameter (that is, for every $\varepsilon>0$, there exists a weak*-open slice $S(x, c, H)$ of $H$ such that $\left.\operatorname{diam}_{A}(S(x, c, H))<\varepsilon\right)$.

In order to characterize weak*-compact $A$-fragmented sets in terms of operator theoretic property (especially, $U_{f}: X \rightarrow L_{\infty}(S, \Sigma, \mu)$ ), we use the following notion concerning subsets of $L_{\infty}(S, \Sigma, \mu)$. This acts as intermediary in our argument.

Definition 7 ([13]). A bounded subset $M$ of $L_{\infty}(S, \Sigma, \mu)$ is said to be equimeasurable if for each $\varepsilon>0$, there is a set $E \in \Sigma$ with $\mu(E)>\mu(S)-\varepsilon$ such that $\left\{f \chi_{E}: f \in M\right\}$ is relatively norm compact in $L_{\infty}(S, \Sigma, \mu)$. 
Now, concerning notions stated in Definitions 2, 3 and 6, we have the following result suggested by Theorems in [6] and [7].

Proposition 2. Let $f: S \rightarrow X^{*}$ be a bounded weak*-measurable function and $A$ a bounded subset of $X$. Suppose that the set $\overline{\mathrm{co}}^{*}\left(T_{f}^{*}(\Delta(E))\right)$ is $A$ weak*-dentable for every $E \in \Sigma^{+}$. Then $f$ has the A-strongly measurable decomposability.

Proof. Since the proof can be given by the same argument as that of the implication (c) $\Rightarrow(d)$ of Theorem in [6] (or, that of Theorem in [7]), it is omitted.

In connection with Definition 7, we note the following result concerning bounded linear operators $U_{f}: X \rightarrow L_{\infty}(S, \Sigma, \mu)$ associated with an $A$-strongly measurable function $f$.

Proposition 3. Let $A$ be a bounded subset of $X$ and $f: S \rightarrow X^{*}$ a bounded weak*-measurable function having the A-strongly measurable decomposability. Then $U_{f}(A)$ is equimeasurable in $L_{\infty}(S, \Sigma, \mu)$.

Proof. Let $h$ be an $A$-strongly measurable function such that $f-h$ is weak*-scalarly null. Then $U_{f}(A)=U_{h}(A)$, and, because of the $A$-strong measurability of $h$, the well-known exhaustion argument assures that for each $n \geqq 1$ there exists a disjoint sequence (possibly finite) $\{E(n, i)\}_{i \geqq 1}$ of $\Sigma^{+}$such that $\mu\left(S \backslash \bigcup_{i \geqq 1} E(n, i)\right)=0$ and $\sup _{x \in A} 0(x \mid h(E(n, i)))<1 / n$ for all $i \geqq 1$. To prove that $U_{h}(A)$ is equimeasurable in $L_{\infty}(S, \Sigma, \mu)$, let $\varepsilon>0$. Then, for every $n \geqq 1$ there exists a natural number $m(n)$ such that $\mu(S \backslash \bigcup\{E(n, i)$ : $1 \leqq i \leqq m(n)\})<\varepsilon / 2^{n}$, and let $E_{n}=\bigcup\{E(n, i): 1 \leqq i \leqq m(n)\}$ for every $n \geqq 1$. Then, for $E=\bigcap_{n \geqq 1} E_{n}$, we have that $\mu(S \backslash E) \leqq \sum_{n \geqq 1} \mu\left(S \backslash E_{n}\right)<\varepsilon$. Furthermore, $\left\{x \circ h \chi_{E}: x \in A\right\}$ is relatively norm compact in $L_{\infty}(S, \Sigma, \mu)$. Indeed, let $\delta>0$, and choose a natural number $k$ with $1 / k<\delta$. Then we have that for every $x \in A$

$$
\begin{aligned}
& \sup \{\operatorname{ess}-0(x \circ h \mid E(k, i) \cap E): 1 \leqq i \leqq m(k)\} \\
& \quad \leqq \sup \{0(x \circ h \mid E(k, i)): 1 \leqq i \leqq m(k)\}<1 / k<\delta .
\end{aligned}
$$

This means the relative norm compactness of $\left\{x \circ h \chi_{E}: x \in A\right\}$ in $L_{\infty}(S, \Sigma, \mu)$. Thus the proof is completed.

Let $A$ be a bounded subset of $X$ and $C=\left\{x_{n}: n \geqq 1\right\}$ a countable subset of $A$. Then, suggested by [3], we wish to consider the bounded linear operator $T_{c}: \ell_{1} \rightarrow X$ given by

$$
T_{c}\left(\left\{a_{n}\right\}_{n \geqq 1}\right)=\sum_{n \geqq 1} a_{n} x_{n}
$$


for every $\left\{a_{n}\right\}_{n \geqq 1} \in \ell_{1}$. Then $T_{c}\left(e_{n}\right)=x_{n}$ for all $n$, where $e_{n}$ denotes the $n$-th unit vector of $\ell_{1}$. Making use of these operators, we can clarify slight directly a relation between $A$-fragmentedness and separability conditions in the following form. We think naturally that this fact has been already known essentially. But we think that it should be expressed once in this form, and it is convenient for us to obtain Proposition 5 which follows Proposition 4.

Proposition 4. Let $A$ be a bounded subset of $X$ and $K$ a weak*-compact subset of $X^{*}$. Then the set $K$ is A-fragmented if and only if for every countable subset $C$ of $A, T_{c}^{*}(K)$ is a separable subset of $\ell_{\infty}$.

Proof. Suppose that $K$ is non- $A$-fragmented. Then there exist a weak*compact subset $D$ of $K$ and an $\varepsilon>0$ such that $\operatorname{diam}_{A}(U \cap D)>\varepsilon$ whenever $U$ is a weak*-open subset with $U \cap D \neq \varnothing$. Hence, replacing the unit ball by a bounded set $A$ in the proof of Proposition 5.6 in [11], we can construct a system $\left\{x(n, i): n=0,1, \ldots ; i=0, \ldots, 2^{n}-1\right\}$ in $A$ and a system $\{U(n, i): n=0,1, \ldots$; $\left.i=0, \ldots, 2^{n}-1\right\}$ of weak*-open subsets such that

(a) $U(n, i) \cap D \neq \varnothing$,

(b) $(U(n+1,2 i) \cap D) \cup(U(n+1,2 i+1) \cap D) \subset U(n, i) \cap D$,

(c) $x^{*} \in U(n+1,2 i)$ and $y^{*} \in U(n+1,2 i+1)$ imply $\left(x(n, i), x^{*}-y^{*}\right) \geqq \varepsilon$ for $n=0,1, \ldots$ and $i=0, \ldots, 2^{n}-1$.

Let $V(n, i)=w^{*}-c l(U(n, i) \cap D)$ (the weak*-closure of $\left.U(n, i) \cap D\right)$. Then we have that

(d) $V(n+1,2 i) \cup V(n+1,2 i+1) \subset V(n, i)$

(e) $x^{*} \in V(n+1,2 i)$ and $y^{*} \in V(n+1,2 i+1)$ imply $\left(x(n, i), x^{*}-y^{*}\right) \geqq \varepsilon$ for $n=0,1, \ldots$ and $i=0, \ldots, 2^{n}-1$.

Let $C=\left\{x(n, i): n=0,1, \ldots ; i=0, \ldots, 2^{n}-1\right\}=\left\{x_{n}: n \geqq 1\right\} \quad$ (Here, for $n=$ $2^{m}+i$ with $m=0,1, \ldots$ and $i=0, \ldots, 2^{m}-1$, define $\left.x_{n}=x(m, i)\right)$ and

$$
\Gamma=\bigcap_{n=0}^{\infty}\left(\bigcup_{i=0}^{2^{n}-1} V(n, i)\right) .
$$

Then, by the same argument as in the proof of the implication (c) $\Rightarrow$ (a) of Theorem 4.2.13 in [2], we easily get that $T_{c}^{*}(\Gamma)$ is not separable, whence $T_{c}^{*}(K)$ is not separable, a contradiction.

Conversely, suppose that there exists a countable subset $C=\left\{x_{n}: n \geqq 1\right\}$ of $A$ such that $T_{c}^{*}(K)$ is not a separable subset of $\ell_{\infty}$. Then the following argument is usual. Choose a weak*-compact subset $B$ of $T_{c}^{*}(K)$ such that $\inf \{\operatorname{diam}(W \cap B): W \cap B \neq \varnothing, W$ is weak*-open $\} \quad(=\varepsilon)>0$. Let $M=K \cap$ $\left(T_{c}^{*}\right)^{-1}(B)$. Then $M$ is a weak*-compact subset of $K$ such that $T_{c}^{*}(M)=B$. Using Zorn's lemma, find a minimal weak*-compact subset $M_{0}$ of $M$ such that $T_{c}^{*}\left(M_{0}\right)=B$. Since $K$ is $A$-fragmented, there exists a weak*-open subset $U$ with $U \cap M_{0} \neq \varnothing$ such that $\operatorname{diam}_{A}\left(U \cap M_{0}\right)<\varepsilon$. Let $W_{0}=\ell_{\infty} \backslash T_{c}^{*}\left(M_{0} \backslash U\right)$. Then it follows from the minimality of $M_{0}$ that $W_{0}$ is a weak*-open subset with 
$W_{0} \cap B \neq \varnothing$, and $W_{0} \cap B \subset T_{c}^{*}\left(U \cap M_{0}\right)$. Hence we have that

$$
\begin{aligned}
\varepsilon & \leqq \operatorname{diam}\left(W_{0} \cap B\right)=\sup _{n \geqq 1} 0\left(e_{n} \mid W_{0} \cap B\right) \leqq \sup _{n \geqq 1} 0\left(e_{n} \mid T_{c}^{*}\left(U \cap M_{0}\right)\right) \\
& =\sup _{n \geqq 1} 0\left(T_{c}\left(e_{n}\right) \mid U \cap M_{0}\right)=\sup _{n \geqq 1} 0\left(x_{n} \mid U \cap M_{0}\right) \leqq \sup _{x \in A} 0\left(x \mid U \cap M_{0}\right) \\
& =\operatorname{diam}_{A}\left(U \cap M_{0}\right)<\varepsilon,
\end{aligned}
$$

which is a contradiction. Thus the proof is completed.

Combining this result with the well-known fact that if $H$ is a separable weak*-compact subset of a dual Banach space, then so is $\overline{\mathrm{co}}^{*}(H)$, we have:

Proposition 5. Let $A$ be a bounded subset of $X$ and $K$ a weak*-compact subset of $X^{*}$. Then the set $K$ is $A$-fragmented if and only if the set $\overline{\mathrm{co}}^{*}(K)$ is A-fragmented.

Proof. This follows immediately from Proposition 4, the remark preceding Proposition 5, and the fact that $T_{c}^{*}\left(\overline{\mathrm{co}}^{*}(K)\right)=\overline{\mathrm{co}}^{*}\left(T_{c}^{*}(K)\right)$ for every countable subset $C$ of $A$.

\section{$\S 3$. Functions Associated with Non- $A$-fragmented Sets and Proof of Theorem}

Let $A$ be a bounded subset of $X$ and $K$ a weak*-compact subset of $X^{*}$. Assume that $K$ is non- $A$-fragmented. Then it follows from the former part of the proof of Proposition 4 that there exist an $\varepsilon>0$, a system $\{x(n, i)$ : $\left.n=0,1, \ldots ; i=0, \ldots, 2^{n}-1\right\} \quad$ in $A$ and a system $\{V(n, i): n=0,1, \ldots$; $\left.i=0, \ldots, 2^{n}-1\right\}$ of non-empty weak ${ }^{*}$-closed subsets of $K$ such that

(a) $V(n+1,2 i) \cup V(n+1,2 i+1) \subset V(n, i)$,

(b) $x^{*} \in V(n+1,2 i)$ and $y^{*} \in V(n+1,2 i+1)$ imply $\left(x(n, i), x^{*}-y^{*}\right) \geqq \varepsilon$ for $n=0,1, \ldots$ and $i=0, \ldots, 2^{n}-1$.

In the following, let $\varepsilon$ be this positive number, $a(n, i)=\inf \left\{\left(x(n, i), x^{*}\right): x^{*} \in\right.$ $V(n+1,2 i)\}$ and $b(n, i)=\sup \left\{\left(x(n, i), x^{*}\right): x^{*} \in V(n+1,2 i+1)\right\}$ for every $(n, i)$. Then it holds that $a(n, i)-b(n, i) \geqq \varepsilon$ for all $(n, i)$.

Now, letting $A_{n}=\bigcup\left\{V(n, 2 i+1): i=0, \ldots, 2^{n-1}-1\right\} \quad$ and $\quad B_{n}=$ $\bigcup\left\{V(n, 2 i): i=0, \ldots, 2^{n-1}-1\right\}$ for every $n \geqq 1,\left(A_{n}, B_{n}\right)_{n \geqq 1}$ is an independent sequence of pairs of weak*-closed subsets of $K$. Then, $\Gamma=\bigcap_{n \geq 1}\left(A_{n} \cup B_{n}\right)$ (Needless to say, this is the same as one in the proof of Proposition 4.) is a nonempty weak*-compact subset of $K$, since $\left(A_{n}, B_{n}\right)_{n \geqq 1}$ is independent. Now, define $\psi: \Gamma \rightarrow \mathscr{P}(N)$ (Cantor space, with its usual compact metric topology) by $\psi\left(x^{*}\right)=\left\{j: A_{j} \ni x^{*}\right\} \in \mathscr{P}(N)$. Then $\psi$ is a continuous surjection and so we have a Radon probability measure $\gamma$ on $\Gamma$ such that $\psi(\gamma)=v$ (the normalized Haar measure if we identify $\mathscr{P}(N)$ with $\left.\{0,1\}^{N}\right)$ and $\{u \circ \psi: u \in$ $\left.L_{1}\left(\mathscr{P}(N), \Sigma_{v}, v\right)\right\}=L_{1}\left(\Gamma, \Sigma_{\gamma}, \gamma\right)$, where $\Sigma_{v}$ (resp. $\Sigma_{\gamma}$ ) is the family of all $v$ (resp. $\gamma$ )-measurable subsets of $\mathscr{P}(N)$ (resp. $\Gamma$ ). Further, consider a function 
$\tau: \mathscr{P}(N) \rightarrow I$ defined by $\tau(J)=\sum\left\{1 / 2^{J}: j \in J\right\}$ for every $J \in \mathscr{P}(N)$. Then $\tau$ is a continuous surjection such that $\tau(v)=\lambda$ and $\left\{v \circ \tau: v \in L_{1}(I, \Lambda, \lambda)\right\}=$ $L_{1}\left(\mathscr{P}(N), \Sigma_{v}, v\right)$. Making use of the lifting theory, we have a weak*-measurable function $h: I \rightarrow \Gamma(\subset D)$ such that

(c) $\rho(f \circ h)(t)=f(h(t))$ for every $f \in C(\Gamma)$ and every $t \in I$,

(d) $\int_{E} f(h(t)) d \lambda(t)=\int_{\psi^{-1}\left(\tau^{-1}(E)\right)} f\left(x^{*}\right) d \gamma\left(x^{*}\right)$

for every $E \in \Lambda$ and every $f \in C(\Gamma)$ (cf. [6]). Here $\rho$ is a lifting on $L_{\infty}(I, \Lambda, \lambda)$. Further we should remark here that $\tau(\psi(\gamma))=\lambda, \psi^{-1}\left(\tau^{-1}(I(n, 2 i))\right) \subset V(n, 2 i)$ and $\psi^{-1}\left(\tau^{-1}(I(n, 2 i+1))\right) \subset V(n, 2 i+1)$ for $n=1,2, \ldots$ and $i=0, \ldots, 2^{n-1}-1$, where $I(n, i)$ denotes an open interval in $I$ given by $I(n, i)=\left(i / 2^{n},(i+1) / 2^{n}\right)$ for every $n \geqq 0$ and $0 \leqq i \leqq 2^{n}-1$.

Concerning this $K$-valued weak*-measurable function $h$ and the system $\left\{x(n, i): n=0,1, \ldots ; i=0, \ldots, 2^{n}-1\right\}$ in $A$ constructed in the case where $K$ is non- $A$-fragmented, we have the following two Propositions 6 and 7, which are fundamental results in our paper. In Proposition 7, the sequence $\left\{x_{n}\right\}_{n \geq 1}$ is given by $x_{n}=x(m, i)$ if $n=2^{m}+i$ for every $m=0,1, \ldots$ and $i=0, \ldots, 2^{m}-1$, and the subset $H$ of $K$ is given by $H=h(I)$.

Proposition 6. The set $U_{h}(A)$ is not equimeasurable in $L_{\infty}(I, A, \lambda)$.

Proof. Suppose that $U_{h}(A)$ is equimeasurable in $L_{\infty}(I, \Lambda, \lambda)$. Then we have an $E \in \Lambda^{+}$such that $\left\{x \circ h \chi_{E}: x \in A\right\}$ is relatively norm compact in $L_{\infty}(I, \Lambda, \lambda)$. Let $\left\{E_{1}, \ldots, E_{m}\right\}$ be a positive measurable partition of $E$. Then, by virtue of Lemma 2 in [5], there exist a natural number $p$ and a finite collection $\left\{i_{1}, \ldots, i_{m}\right\}$ of non-negative integers such that $0 \leqq 2 \cdot i_{k}<2^{p}-1, E_{k} \cap$ $I\left(p, 2 \cdot i_{k}\right) \in \Lambda^{+}$and $E_{k} \cap I\left(p, 2 \cdot i_{k}+1\right) \in \Lambda^{+}$for $k=1, \ldots, m$. Let $F_{k}=E_{k} \cap$ $I\left(p, 2 \cdot i_{k}\right)$ and $G_{k}=E_{k} \cap I\left(p, 2 \cdot i_{k}+1\right)$ for $k=1, \ldots, m$. Then we have that for every $k$

$$
\begin{aligned}
\text { ess- } 0 & \left.x\left(p-1, i_{k}\right) \circ h \mid E_{k}\right) \\
= & \operatorname{ess-sup}_{t \in E_{k}}\left(x\left(p-1, i_{k}\right), h(t)\right)-\text { ess- inf } \operatorname{ti}_{k}\left(x\left(p-1, i_{k}\right), h(t)\right) \\
\geqq & \left\{\int_{F_{k}}\left(x\left(p-1, i_{k}\right), h(t)\right) d \lambda(t)\right\} / \lambda\left(F_{k}\right) \\
& -\left\{\int_{G_{k}}\left(x\left(p-1, i_{k}\right), h(t)\right) d \lambda(t)\right\} / \lambda\left(G_{k}\right) \\
= & \left\{\int_{\psi^{-1}\left(\tau^{-1}\left(F_{h}\right)\right)}\left(x\left(p-1, i_{k}\right), x^{*}\right) d \gamma\left(x^{*}\right)\right\} / \lambda\left(F_{k}\right) \\
& -\left\{\int_{\psi^{-1}\left(\tau^{-1}\left(G_{k}\right)\right)}\left(x\left(p-1, i_{k}\right), x^{*}\right) d \gamma\left(x^{*}\right)\right\} / \lambda\left(G_{k}\right) \\
\geqq & a\left(p-1, i_{k}\right)-b\left(p-1, i_{k}\right) \geqq \varepsilon .
\end{aligned}
$$


Hence we get that $\left\{x \circ h \chi_{E}: x \in A\right\}$ is not relatively norm compact in $L_{\infty}(I, \Lambda, \lambda)$, which is a contradiction. Thus the proof is completed.

Proposition 7. The function $c_{H}$ is nowhere $\Psi$-uniformly Gateaux differentiable in $X$, where $\Psi=\left\{x_{n}: n \geqq 1\right\}$.

Proof. Take any point $x$ of $X$. Let $M=\overline{\operatorname{co}}^{*}\left(T_{h}^{*}(\Delta(I))\right)$, and consider a family of weak*-open slices: $\{S(x, \varepsilon / 3 n, M): n \geqq 1\}$. Then we have that for every $n \geqq 1$

$$
\begin{aligned}
S(x, \varepsilon / 3 n, M) & =\left\{x^{*} \in M:\left(x, x^{*}\right)>\sup _{z^{*} \in M}\left(x, z^{*}\right)-\varepsilon / 3 n\right\} \\
& =\left\{x^{*} \in M:\left(x, x^{*}\right)>\underset{t \in I}{\operatorname{ess}-\sup }(x, h(t))-\varepsilon / 3 n\right\} \\
& =\left\{x^{*} \in M:\left(x, x^{*}\right)>c_{H}(x)-\varepsilon / 3 n\right\} .
\end{aligned}
$$

So, letting $E_{n}=\left\{t \in I:(x, h(t))>c_{H}(x)-\varepsilon / 3 n\right\}$, we know that $E_{n} \in \Lambda^{+}$and $h\left(E_{n}\right) \subset S(x, \varepsilon / 3 n, M)$ for every $n$. Hence, by Lemma 2 (and its proof) in [5], there exist a strictly increasing sequence $\left\{p_{n}\right\}_{n \geqq 1}$ of natural numbers and a sequence $\left\{i_{n}\right\}_{n \geqq 1}$ of non-negative integers such that $0 \leqq 2 \cdot i_{n}<2^{p_{n}}-1, E_{n} \cap$ $I\left(p_{n}, 2 \cdot i_{n}\right) \in \Lambda^{+}$and $E_{n} \cap I\left(p_{n}, 2 \cdot i_{n}+1\right) \in \Lambda^{+}$for every $n \geqq 1$. Let $F_{n}=E_{n} \cap$ $I\left(p_{n}, 2 \cdot i_{n}\right)$ and $G_{n}=E_{n} \cap I\left(p_{n}, 2 \cdot i_{n}+1\right)$, and define $u_{n}^{*}=T_{h}^{*}\left(\chi_{F_{n}} / \lambda\left(F_{n}\right)\right)$ and $v_{n}^{*}=T_{h}^{*}\left(\chi_{G_{n}} / \lambda\left(G_{n}\right)\right)$ for every $n \geqq 1$. Then we have that for every $n$

$(\alpha)\left(x, u_{n}^{*}\right)>c_{H}(x)-\varepsilon / 3 n$ and $\left(x, v_{n}^{*}\right)>c_{H}(x)-\varepsilon / 3 n$,

( $\beta) \quad\left(y_{n}, u_{n}^{*}-v_{n}^{*}\right) \geqq \varepsilon \quad\left(\right.$ Here,,$y_{n}=x\left(p_{n}-1, i_{n}\right)$, and so, $\left\{y_{n}\right\}_{n \geqq 1}$ is a subsequence of $\left.\left\{x_{n}\right\}_{n \geq 1}\right)$,

$(\gamma) \quad c_{H}\left(x+y_{n} / n\right) \geqq\left(x+y_{n} / n, u_{n}^{*}\right)$ and $c_{H}\left(x-y_{n} / n\right) \geqq\left(x-y_{n} / n, v_{n}^{*}\right)$.

Indeed, we have that

$$
\begin{aligned}
\left(x, u_{n}^{*}\right) & =\left(x, T_{h}^{*}\left(\chi_{F_{n}} / \lambda\left(F_{n}\right)\right)\right) \\
& =\left\{\int_{F_{n}}(x, h(t)) d \lambda(t)\right\} / \lambda\left(F_{n}\right)>c_{H}(x)-\varepsilon / 3 n,
\end{aligned}
$$

since $h\left(F_{n}\right) \subset S(x, \varepsilon / 3 n, M)$. Similarly, $\left(x, v_{n}^{*}\right)>c_{H}(x)-\varepsilon / 3 n$. Thus we have $(\alpha)$. Also we can prove $(\beta)$ as follows. In virtue of the fact preceding Proposition 6, we have that for every $n$

$$
\begin{aligned}
& \left(y_{n}, u_{n}^{*}-v_{n}^{*}\right) \\
& \quad=\left(y_{n}, T_{h}^{*}\left(\chi_{F_{n}} / \lambda\left(F_{n}\right)\right)\right)-\left(y_{n}, T_{h}^{*}\left(\chi_{G_{n}} / \lambda\left(G_{n}\right)\right)\right) \\
& \quad=\left(x\left(p_{n}-1, i_{n}\right), T_{h}^{*}\left(\chi_{F_{n}} / \lambda\left(F_{n}\right)\right)\right)-\left(x\left(p_{n}-1, i_{n}\right), T_{h}^{*}\left(\chi_{G_{n}} / \lambda\left(G_{n}\right)\right)\right)
\end{aligned}
$$




$$
\begin{aligned}
= & \left\{\int_{F_{n}}\left(x\left(p_{n}-1, i_{n}\right), h(t)\right) d \lambda(t)\right\} / \lambda\left(F_{n}\right) \\
& -\left\{\int_{G_{n}}\left(x\left(p_{n}-1, i_{n}\right), h(t)\right) d \lambda(t)\right\} / \lambda\left(G_{n}\right) \\
= & \left\{\int_{\psi^{-1}\left(\tau^{-1}\left(F_{n}\right)\right)}\left(x\left(p_{n}-1, i_{n}\right), x^{*}\right) d \gamma\left(x^{*}\right)\right\} / \lambda\left(F_{n}\right) \\
& -\left\{\int_{\psi^{-1}\left(\tau^{-1}\left(G_{n}\right)\right)}\left(x\left(p_{n}-1, i_{n}\right), x^{*}\right) d \gamma\left(x^{*}\right)\right\} / \lambda\left(G_{n}\right) \\
\geqq & a\left(p_{n}-1, i_{n}\right)-b\left(p_{n}-1, i_{n}\right) \geqq \varepsilon .
\end{aligned}
$$

As to $(\gamma)$, we have that for every $n$

$$
\begin{aligned}
c_{H}\left(x+y_{n} / n\right) & =\sup _{t \in I}\left(x+y_{n} / n, h(t)\right) \\
& \geqq\left\{\int_{F_{n}}\left(x+y_{n} / n, h(t)\right) d \lambda(t)\right\} / \lambda\left(F_{n}\right)=\left(x+y_{n} / n, u_{n}^{*}\right) .
\end{aligned}
$$

Similarly, $c_{H}\left(x-y_{n} / n\right) \geqq\left(x-y_{n} / n, v_{n}^{*}\right)$. Hence, making use of $(\alpha),(\beta)$ and $(\gamma)$, we get that for every $n$

$$
\begin{aligned}
c_{H}(x & \left.+y_{n} / n\right)+c_{H}\left(x-y_{n} / n\right)-2 \cdot c_{H}(x) \\
& >\left(x+y_{n} / n, u_{n}^{*}\right)+\left(x-y_{n} / n, v_{n}^{*}\right)-\left\{\left(x, u_{n}^{*}+v_{n}^{*}\right)+2 \varepsilon / 3 n\right\} \\
& =\left(y_{n}, u_{n}^{*}-v_{n}^{*}\right) / n-2 \varepsilon / 3 n \geqq \varepsilon / 3 n .
\end{aligned}
$$

So we have that $\left\{c_{H}\left(x+y_{n} / n\right)+c_{H}\left(x-y_{n} / n\right)-2 \cdot c_{H}(x)\right\} /(1 / n)>\varepsilon / 3$ for every $n$, which implies that $c_{H}$ is not $\Psi$-uniformly Gateaux differentiable at $x$ by virtue of Proposition 1. Thus the proof is completed.

Remark 1. By the same way as above, we easily get the following results.

(1) The tree $\left\{2^{n} \alpha_{h}(I(n, i)): n=0,1, \ldots ; i=0, \ldots, 2^{n}-1\right\}$ (associated with h) satisfies that for every $n \geqq 1$ and $i$ with $0 \leqq i \leqq 2^{n-1}-1, q_{A}\left(2^{n} \alpha_{h}(I(n, 2 i))-\right.$ $\left.2^{n} \alpha_{h}(I(n, 2 i+1))\right) \geqq \varepsilon$.

(2) The usual dyadic martingale $\left(h_{n}, \Lambda_{n}\right)_{n \geqq 1}$ associated with $h$ satisfies that

$$
\inf _{n \geqq 1} \int_{I} q_{A}\left(h_{n}(t)-h_{n+1}(t)\right) d \lambda(t) \geqq \varepsilon / 2>0
$$

(3) The set $\overline{\mathrm{co}}^{*}\left(T_{h}^{*}(\Delta(E))\right)$ is not $A$-weak*-dentable for every $E \in \Lambda^{+}$. For instance, to show (1), we estimate $q_{A}\left(2^{n} \alpha_{h}(I(n, 2 i))-2^{n} \alpha_{h}(I(n, 2 i+1))\right)$ for every $n \geqq 1$ and $i$ with $0 \leqq i \leqq 2^{n-1}-1$. That is, we have that 


$$
\begin{aligned}
q_{A}\left(2^{n} \alpha_{h}(I(n, 2 i))-2^{n} \alpha_{h}(I(n, 2 i+1))\right) \\
\geqq 2^{n} \cdot\left(x(n-1, i), \alpha_{h}(I(n, 2 i))-\alpha_{h}(I(n, 2 i+1))\right) \\
=2^{n}\left\{\int_{I(n, 2 i)}(x(n-1, i), h(t)) d \lambda(t)-\int_{I(n, 2 i+1)}(x(n-1, i), h(t)) d \lambda(t)\right\} \\
=2^{n}\left\{\int_{\psi^{-1}\left(\tau^{-1}(I(n, 2 i))\right)}\left(x(n-1, i), x^{*}\right) d \gamma\left(x^{*}\right)\right. \\
\left.\quad-\int_{\psi^{-1}\left(\tau^{-1}(I(n, 2 i+1))\right)}\left(x(n-1, i), x^{*}\right) d \gamma\left(x^{*}\right)\right\} \\
\geqq a(n-1, i)-b(n-1, i) \geqq \varepsilon .
\end{aligned}
$$

Now we are ready to give a proof of Theorem.

Proof of Theorem. (1) $\Rightarrow(2)$. This is clear.

$(2) \Rightarrow(3)$. This follows from Proposition 3.

$(3) \Rightarrow(4)$. This follows from Proposition 6.

(4) $\Rightarrow(5)$. This follows from Proposition 5 .

$(5) \Rightarrow(6)$. The proof of this part can be given by the same argument as in the corresponding one of Theorem 4.2.13 in [2]. That is, we may replace the norm $\|\cdot\|$ by the seminorm $q_{A}$ in that place.

$(6) \Rightarrow(1)$. This follows from Proposition 2, since $\overline{\mathrm{co}}^{*}\left(T_{h}^{*}(\Delta(E))\right)$ is a weak*-compact convex subset of $\overline{\mathrm{co}}^{*}(K)$ for every weak*-measurable function $f: S \rightarrow K$ and every $E \in \Sigma^{+}$.

$(6) \Rightarrow(7)$. This can be shown by the same argument as in the proof of Theorem 3.14 and Proposition 3.15 of [3].

$(7) \Rightarrow(8)$. This follows immediately, since $\partial c_{H}(x) \subset \overline{\mathrm{co}}^{*}(K)$ for every subset $H$ of $K$ and every $x \in X$.

$(8) \Rightarrow(4)$. This follows from Proposition 7

\section{§4. Remarks on Theorem}

Let us give some remarks on Theorem.

Remark 2. As stated above, Fitzpatrick has defined separably related sets in $X^{*}$ as follows: Let $K$ be a weak*-compact subset of $X^{*}$ and $A$ a bounded subset of $X$. Then the set $K$ is said to be separably related to $A$ if for every countable subset $C$ of $A$, the set $K$ is separable in the seminormed space $\left(X^{*}, q_{c}\right)$. Well, since we can easily get that $K$ is separably related to $A$ if and only if for every countable subset $C$ of $A, T_{c}^{*}(K)$ is separable in $\ell_{\infty}$, each of the statements $(1) \sim(8)$ (especially, (1), (2) and (8)) in Theorem is equivalent to that $K$ is separably related to $A$. 
Remark 3. Following Stegall [13], we say that a bounded subset $A$ of $X$ is a GSP set if for every $(S, \Sigma, \mu)$ and every bounded linear operator $U: X \rightarrow$ $L_{\infty}(S, \Sigma, \mu), U(A)$ is equimeasurable in $L_{\infty}(S, \Sigma, \mu)$. Then, setting $K=B\left(X^{*}\right)$ in Theorem, we know that $A$ is a GSP set if and only if $B\left(X^{*}\right)$ has the $A$-RNP.

Remark 4. We have studied the properties of weak*-compact $A$ fragmented sets $K$ from our standpoint to deal with a weak*-compact $A$ fragmented set itself and to clarify its structure directly (that is, without using results already known in the case where $A=B(X)$ or $K=B\left(X^{*}\right)$ ). So, in comparison with other studies of such topics treated in Theorem, the major merit of our approach and argument may be that we can immediately get various well-known characterizations of RN sets, GSP sets and so on, by an appropriate choice of the sets $A$ and $K$ in Theorem.

\section{References}

[1] Asplund, E. and Rockafellar, R. T., Gradient of convex functions, Trans. Amer. Math. Soc., 139 (1969), 443-467.

[2] Bourgin, R. D., Geometric aspects of convex sets with the Radon-Nikodym property, Lecture Notes in Math., 993 (1983), Springer.

[3] Fitzpatrick, S. P., Separably related sets and the Radon-Nikodym property, Illinois J. Math., 29 (1985), 229-247.

[4] Jayne, J. E. and Rogers, C. A., Borel selectors for upper semicontinuous set-valued maps, Acta Math., 155 (1985), 41-79.

[5] Matsuda, M., A characterization of Pettis sets in terms of the Bourgain property, Math. Japon., 41 (1995), 433-439.

[6] _- On localized weak precompactness in Banach spaces, Publ. RIMS, Kyoto Univ., 32 (1996), 473-491.

[7] - On certain decomposition of bounded weak*-measurable functions taking their ranges in dual Banach spaces, Hiroshima Math. J., 27 (1997), 429-437.

[8] — - On localized weak precompactness in Banach spaces II, Hiroshima Math. J., 28 (1998), 399-418.

[9] - A remark on localized weak precompactness in Banach spaces, Comment. Math. Univ. Carolinae, 40 (1999), 271-276.

[10] Michael, E. and Namioka I., Barely continuous functions, Bull. Acad. Polon. Sci. Ser. Math. Astronom. Phys., 24 (1976), 889-892.

[11] Phelps, R. R., Convex functions, monotone operators and differentiability, Lecture Notes in Math., 1364 (1989), Springer.

[12] Reynov, O. I., On a class of Hausdorff compacts and GSG Banach spaces, Studia Math., 71 (1981), 113-126.

[13] Stegall, C., The Radon-Nikodym property in conjugate Banach spaces. II, Trans. Amer. Math. Soc., 264 (1981), 507-519. 
